DOI 10. 18307/2016. 0621

(c) 2016 by Journal of Lake Sciences

\title{
不同鱼类对沉水植物生长的影响"
}

\author{
王晓平 ${ }^{1}$, 王玉兵 ${ }^{1}$,杨桂军 ${ }^{1 * *}$, 秦伯强 ${ }^{2}$, 杨宏伟 $^{2}$ \\ (1: 江南大学环境与土木工程学院,无锡 214122) \\ (2: 中国科学院南京地理与湖泊研究所湖泊与环境国家重点实验室,南京 210008)
}

摘 要: 沉水植物是富营养水体生态修复的重要组成部分, 鱼类是影响沉水植物生长的重要因素之一. 为了解鱼类对沉 水植物生长的影响, 研究 3 种鱼 (草鱼 (Ctenopharyngodon idellus)、鲫(Carassius auratus)、鳊 (Parabramis pekinensis)) 对 4 种 沉水植物 (苦草 (Vallisneria natans)、金鱼藻 (Ceratophyllum demersum)、伊乐藻 (Elodea nuttalli)、马来眼子菜 (Potamogeton malaianus) ) 生长的影响. 结果显示, 对照组、草鱼组和鳊组沉水植物生物量分别下降 19.2\%、82.1\%和 $16.6 \%$, 而鲫组沉水 植物生物量却增长 $12.5 \% .4$ 种沉水植物中, 金鱼藻生物量增长最高, 其中对照组、鳊组和鲫组中金鱼藻生物量分别增长 $171.1 \% 、 113.9 \%$ 和 $265.5 \%$, 而草鱼组金鱼藻生物量却下降 $24.4 \%$. 草鱼、鳊、鲫都能有效抑制沉水植物的竞争者水绵的生 长. 该研究结果在利用沉水植物修复富营养水体过程中对鱼类的管理具有重要的参考意义.

关键词: 鱼类;沉水植物; 摄食作用; 水绵

\section{The effects of different fish species on growth of submerged macrophytes}

\author{
WANG Xiaoping $^{1}$, WANG Yubing ${ }^{1}$, YANG Guijun ${ }^{1 * *}$, QIN Boqiang ${ }^{2}$ \& YANG Hongwei $^{2}$ \\ (1: Environment and Civil Engineering School, Jiangnan University, Wuxi 214122, P.R. China) \\ (2: State Key Laboratory of Lake Science and Environment, Nanjing Institute of Geography and Limnology, Chinese Academy \\ of Sciences, Nanjing 210008, P.R.China)
}

Abstract: Submerged macrophyte is an important component in ecological restoration of eutrophic waters. Fish is one of the important factors affecting the growth of submerged macrophytes. In the present study, the effects of three fish species(Ctenopharyngodon idellus, Parabramis pekinensis and Carassius auratus) on growth of four submerged macrophytes (Vallisneria natans, Ceratophyllum demersum, Elodea nuttalli and Potamogeton malaianus) were studied. Results showed that the decline rates of submerged macrophytes biomass were $19.2 \%, 82.1 \%$ and $16.6 \%$ in controls, $C$. idellus and $P$. pekinensis, respectively. However, submerged macrophytes biomass increased $12.5 \%$ in the $C$. auratus treatments. The rate of increase of Ceratophyllum demersum biomss was the highest among the four submerged macrophytes. The rate of increase of $C$. demersum biomass in controls, $P$. pekinensis and $C$. auratus treatments were $171.1 \%, 113.9 \%$ and $265.5 \%$, respectively, but that was $-24.4 \%$ in C. idellus treatments. Moreover, all the three fish species inhibited the growth of Spirogyra which is one of the competitors of submerged macrophytes. The results of this study could be used for the fish management during the restoration of submerged macrophytes in eutrophic waters.

Keywords: Fish; submerged macrophytes; feeding behavior; Spirogyra

作为主要初级生产者, 沉水植物是水生生态系统中重要的结构和功能元素, 在富营养水体生态修复过 程中发挥着维护生态完整性与稳定性、净化水质、抑制藻类生长等重要作用 ${ }^{[1-4]}$. 沉水植物的生长受多种因 子的影响,包括营养盐、动物的牧食、与浮游植物和附着藻类之间的竞争等 ${ }^{[5-6]}$. 鱼类是影响沉水植物生长的 重要因子之一. 目前关于鱼类对沉水植物的生长研究主要集中在草食性鱼类,尤其是草鱼 (Ctenopharyngodon

* 国家水体污染控制与治理科技重大专项 ( 2012ZX07503-002,2012ZX07101-013-03) 和国家自然科学基金重点项目 (41230744) 联合资助. 2015-10-19 收稿; 2016-05-05 收修改稿. 王晓平 (1990 ), 男, 硕士研究生; E-mail: 351159746@ qq.com.

** 通信作者;E-mail: yanggj1979@163.com. 
idellus), 研究表明, 草鱼的大小 ${ }^{[7]}$ 、密度 ${ }^{[8]}$ 以及环境因子如水温和 $\mathrm{pH}$ 值都会影响草鱼对沉水植物的摄食作 用 ${ }^{[9]}$. 而有关其它鱼类对沉水植物的生长的研究国内外较少报道 ${ }^{[10-11]}$. 沉水植被恢复过程中, 草食性鱼类 特别是草鱼的摄食影响很大, 有时甚至出现了毁灭性破坏 ${ }^{[7]}$. 此外, 过量地放养草食性鱼类导致湖泊大型 水生植物群落严重破坏, 最终导致系统退化的现象也普遍存在 ${ }^{[5]}$. 在利用沉水植物修复富营养水体过程中, 如何管理好原水体中的鱼类以及放养何种鱼类, 对沉水植物的生长与恢复具有重要的影响. 因此, 了解不同 鱼类对沉水植物的生长影响对富营养水体修复的管理具有重要的参考价值.

苦草 (Vallisneria natans)、金鱼藻 (Ceratophyllum demersum)、伊乐藻 (Elodea nuttalli)、马来眼子菜 (Potamogeton malaianus) 皆是许多浅水湖泊的常见沉水植物, 太湖中也普遍存在 ${ }^{[12]}$, 并常作为湖泊沉水植被修复 的重要种类 ${ }^{[13]}$. 草鱼、鲫 (Carassius auratus)、鳊(Parabramis pekinensis) 是我国重要的经济鱼类,在许多湖泊、 水库都有放养, 是我国许多水体的优势种类 ${ }^{[14]}$. 草鱼、鳊皆属于草食性鱼类, 而鲫属杂食性鱼类,食物包括 浮游动植物、附着藻类、大型水生植物等. 为了解不同鱼类对富营养水体沉水植物生长的影响, 本文研究 3 种鱼类 (草鱼、鲫、鳊) 对 4 种沉水植物 (苦草、金鱼藻、伊乐藻、马来眼子菜) 生长的影响, 为利用沉水植物进 行富营养水体生态修复以及管理提供科学依据.

\section{1 材料与方法}

\section{1 实验设计}

实验于 2014 年 5-6 月在江南大学进行, 实验用 水箱为 $150 \mathrm{~L}$ 的透明聚乙烯水箱 $(0.63 \mathrm{~m} \times 0.42 \mathrm{~m} \times$ $0.55 \mathrm{~m})$, 实验用水为自来水, 实验时加人自来水 100 $\mathrm{L}\left(\right.$ 经测量计算水深为 $40 \mathrm{~cm}$, 面积为 $0.26 \mathrm{~m}^{2}$ ), 然后 在阳光下放置 $7 \mathrm{~d}$,达到去除去水中氯气的目的.实验 箱无底泥, 但铺有 $5 \mathrm{~cm}$ 厚的沙子, 用于固定沉水植 物.沉水植物 (马来眼子菜、金鱼藻、伊乐藻、苦草) 来 自于江南大学校园内的小蟙河,鱼类 (草鱼、鳊、鲫) 购自于常州水产养殖场. 实验前将小䗑河采集的沉水 植物种植在水箱中 (表 1), 待水箱中水草稳定生长一 周后放人指定鱼类. 实验分为 4 组, 即对照组、草鱼 组、鳊组和鲫组 (表 2), 每组 3 个重复,共 12 个水箱. 调制实验用水的总氮 $(\mathrm{TN})$ 起始浓度为 $5 \mathrm{mg} / \mathrm{L}$, 总磷 ( TP) 起始浓度为 $0.25 \mathrm{mg} / \mathrm{L}$ (根据太湖梅梁湾多年平 均值, 其中 TN 和 TP 浓度分别用 $\mathrm{NaNO}_{3}$ 和 $\mathrm{K}_{2} \mathrm{HPO}_{4}$. $3 \mathrm{H}_{2} \mathrm{O}$ 来配制). 实验期间平均水温为 $24.6^{\circ} \mathrm{C}$, 为了保 证鱼类有充足的氧气, 充氧装置连接到每个水箱中. 整个实验在露天环境下进行,水箱上方无遮阳篷,但

表 1 实验用沉水植物

Tab.1 The submerged macrophytes used in the experiment

\begin{tabular}{lcc}
\hline 沉水植物 & 长度 $/ \mathrm{cm}$ & 生物量 $/\left(\mathrm{g} / \mathrm{m}^{2}\right)$ \\
\hline 马来眼子菜(种苗) & $32.5 \pm 5.6$ & $152.6 \pm 11.4$ \\
金鱼藻(茎尖) & $16.8 \pm 3.4$ & $54.1 \pm 3.7$ \\
伊乐藻(茎尖) & $15.8 \pm 3.7$ & $56.8 \pm 3.7$ \\
苦草(种苗) & $12.1 \pm 2.6$ & $83.3 \pm 0.3$ \\
\hline
\end{tabular}

表 2 实验用鱼

Tab.2 The fishes used in the experiment

\begin{tabular}{lcccc}
\hline 鱼类 & 尾数 & $\begin{array}{c}\text { 长度/ } \\
\mathrm{cm}\end{array}$ & $\begin{array}{c}\text { 规格/ } \\
(\mathrm{g} / \text { ind. })\end{array}$ & $\begin{array}{c}\text { 生物量/ } \\
\left(\mathrm{g} / \mathrm{m}^{2}\right)\end{array}$ \\
\hline 草鱼组 & 2 & $16 \pm 1$ & $81 \pm 3$ & $1600 \pm 40$ \\
鳊组 & 4 & $14 \pm 1$ & $31 \pm 2$ & $1240 \pm 32$ \\
鲫组 & 1 & $18 \pm 1$ & $125 \pm 5$ & $1250 \pm 35$ \\
\hline
\end{tabular}

覆盖有孔眼为 $2 \mathrm{~cm}$ 的纱网布, 以防鱼类跳出.

实验期间每 4 天进行 1 次采样 (采样时间为 $14: 00$ ), 每次采集水样 $250 \mathrm{ml}$, 分析 TN、TP、总溶解性氮 (DTN) 、总溶解性磷 (DTP)、叶绿素 a (Chl.a) 浓度. 同时, 实验初始 (表 1) 和实验结束后分别测定沉水植物 的湿重与长度. 用碱性过硫酸钾消解紫外分光光度法测定 TN D DTN 浓度, 用锄酸铵分光光度法测定 TP、DTP 浓度 ${ }^{[15]}$. 采用丙酮萃取分光光度法测定水中 Chl.a 浓度 ${ }^{[16]}$. 在整个实验过程中, 每天测定水温, 观察水箱内 不同鱼类的生长情况, 如有鱼死亡, 则补充相同大小、质量的同种鱼. 为了保证实验过程中水箱水体积的稳 定, 每次采样后每个水箱中加人适量的蒸馏水, 以补充水箱因蒸发和采样损失的水量. 因实验期间草鱼组对 沉水植物摄食很快, 实验进行到 $12 \mathrm{~d}$ 时草鱼组沉水植物几乎被摄食完, 为了避免实验继续下去会产生较大 误差, 所以整个实验共进行了 $12 \mathrm{~d}$. 实验结束时, 称量水体中水生植物的湿重, 称量时取沉水植物和水绵, 用 蒸馏水洗净, 沥干 $5 \mathrm{~min}$, 用滤纸吸水后放人电子天平称量, 取 3 次平均值为水生植物湿重. 


\section{2 数据处理与分析统计}

不同处理组数据采用单因素方差分析 (One-way ANOVA), 并应用 Tukey's 多重两两比较平均数之间的 差异显著性, 数据分析采用 SPSS 18.0 软件.

\section{2 结果}

\section{1 不同鱼类对沉水植物生长的影响}

实验结束时, 草鱼组中的沉水植物总生物量明显低于鲫组、鳊组和对照组, 平均为 $63.5 \mathrm{~g} / \mathrm{m}^{2}$, 鳊组和对 照组总生物量分别为 297.3 和 $296.2 \mathrm{~g} / \mathrm{m}^{2}$, 鲫组中的沉水植物总生物量最高, 为 $401.1 \mathrm{~g} / \mathrm{m}^{2}$ (图 1). 实验结束 时, 对照组、鳊组和草鱼组中的沉水植物总生物量都有所下降, 分别下降 $19.2 \% 、 16.6 \%$ 和 $82.1 \%$, 呈现出负增 长趋势, 而鲫组中沉水植物总生物量增加, 增加 $12.5 \%$; 草鱼组、鲫鱼组与对照组沉水植物总生物量存在显著 差异 $(P<0.05)$. 因此草鱼和鳊的存在不利于沉水植物的生长, 而鲫的存在有利于沉水植物的生长与恢复.

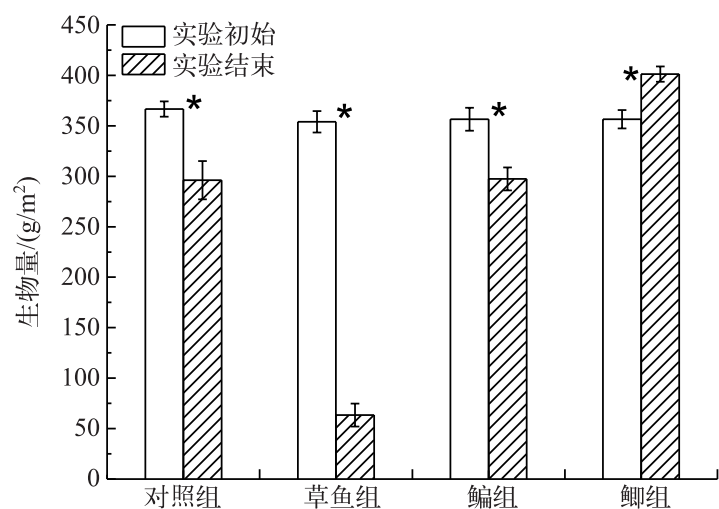

图 1 不同鱼类对沉水植物总生物量的影响 ( $*$ 表示 $P<0.05$, 实验初始和实验结束差异显著;下同 $)$

Fig. 1 The effect of different fishes on total biomass of submerged macrophytes

实验结束时各实验组马来眼子菜总生物量都 比实验初始时明显减少, 表明 3 种鱼类都摄食马来 眼子菜 (图 2). 3 个实验组中, 马来眼子菜的损失量 存在显著差异 $(P<0.05)$. 实验结束时, 草鱼组中马 来眼子菜生物量下降了 $91.8 \%$, 明显高于鳊组 ( $34.2 \%)$ 和鲫组 ( $53.5 \%)$, 对照组中马来眼子菜的 生物量下降了 $0.8 \%$, 基本未变化. 除草鱼组外,其 它 3 组金鱼藻生物量在实验结束时都较实验初始 时明显增加, 对照组、鳕组和鲫组生物量分别增加 $171.1 \% 、 113.9 \%$ 和 $265.6 \%$, 草鱼组生物量下降 $24.4 \%$.

对照组和草鱼组中的伊乐藻生物量都明显下 降, 分别下降 74.8\% 和 100\% (图 2). 鳊组中伊乐 藻生物量下降了 $39.1 \%$, 而鲫组中伊乐藻生物量 变化较小, 基本未发生变化. 与伊乐藻相似, 鲫组 中苦草生物量不降反升, 苦草生物量增长 $19.3 \%$, 而草鱼组与鳊组苦草生物量都有所下降, 鳊组苦 草生物量下降 $10.1 \%$, 而草鱼组生物量下降 $85.1 \%$, 对照组中苦草生物量变化较小, 表明不同鱼类对沉水植 物的摄食具有明显的选择性. 4 种沉水植物生物量变化表明, 草鱼对于沉水植物的摄食偏好顺序为伊乐藻> 马来眼子菜>苦草>金鱼藻, 鳊的摄食偏好顺序为伊乐藻>马来眼子菜>苦草>金鱼藻, 鲫的摄食偏好顺序为 马来眼子菜>伊乐藻 >苦草 $>$ 金鱼藻. 4 种沉水植物中, 金鱼藻生物量增加最多, 表明 3 种鱼都相对不喜欢摄 食金鱼藻.

在实验过程中, 对照组和实验组中有大量的水绵产生, 其中对照组中的水绵呈现出快速增长的趋势, 在 整个实验过程中共产生 $230.0 \mathrm{~g}$, 生长速率约为 $0.2 \mathrm{~g} /(\mathrm{L} \cdot \mathrm{d})$, 相较于对照组, 实验组中水绵的生物量差异较 小, 当实验结束时, 鳊组和鲫组中共产生 27.1 和 $26.7 \mathrm{~g}$ 水绵,生物量分别为 104.2 和 $102.6 \mathrm{~g} / \mathrm{m}^{2}$, 明显小于对 照组 $\left(884.6 \mathrm{~g} / \mathrm{m}^{2}\right)$. 而草鱼组中产生的水绵全部被草鱼主动摄食. 可见, 3 种鱼类都能够通过主动摄食水绵 来抑制水绵的生长, 其中草鱼的抑制能力最强.

\section{2 不同鱼类对水中营养盐的影响}

实验过程中, 草鱼组、鳊组和鲫组 TN 浓度经历了先小幅下降、然后上升、实验结束时再快速下降的过 程. 实验结束时所有实验组 TN 浓度都高于对照组, 对照组、草鱼组、鳊组和鲫组 TN 浓度分别为 2.7、2.9、4.1 和 $3.4 \mathrm{mg} / \mathrm{L}$. 其中鳊组、鲫组与对照组 TN 浓度均存在显著差异 $(P<0.05)$, 草鱼组与对照组未存在显著差异 $(P>0.05$ ) (图 3A).

除了鳊组外, 其它实验组和对照组 TP 浓度经历先快速下降, 然后上升, 实验结束时再次下降的过程 (图 


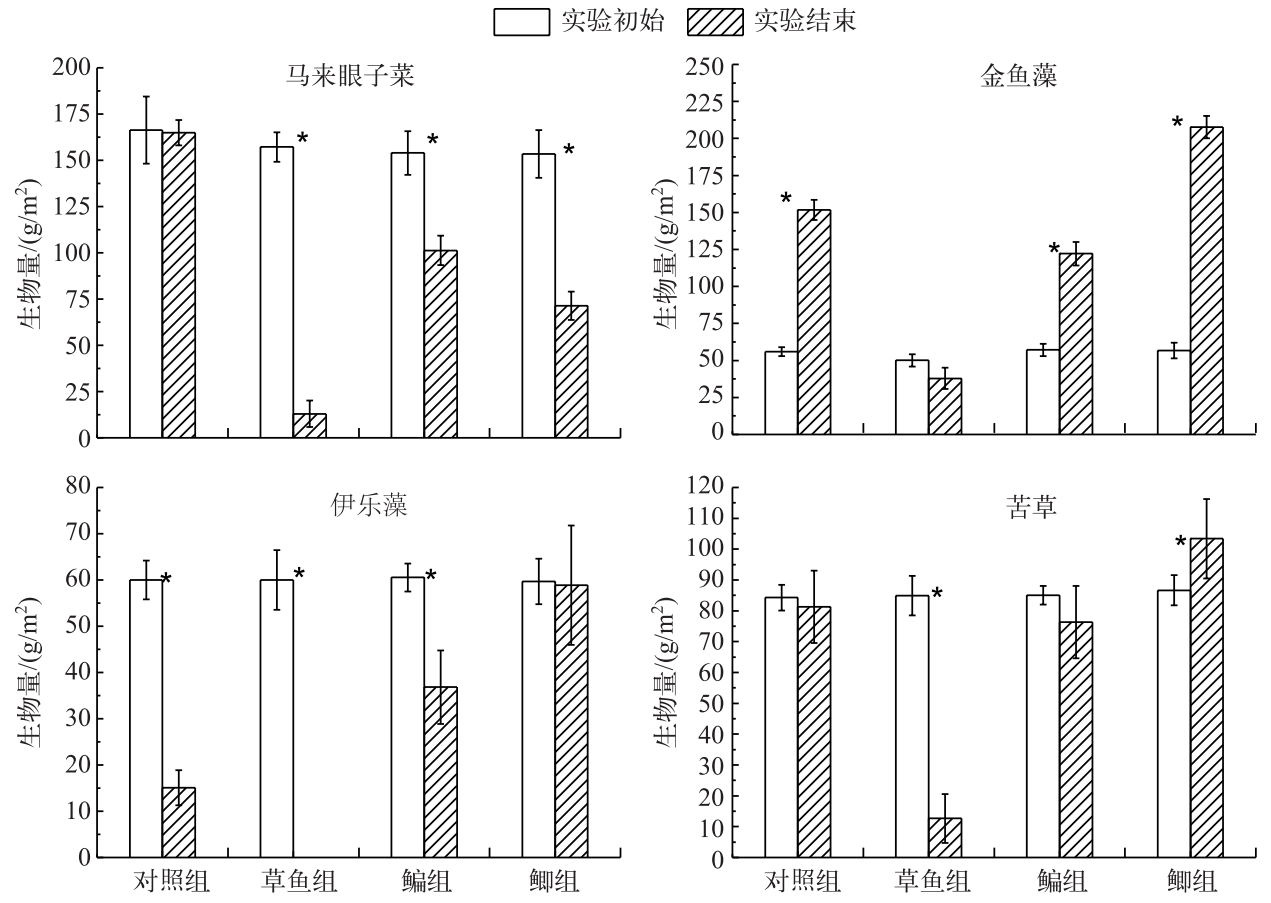

图 2 不同鱼类对不同种类沉水植物生物量的影响

Fig. 2 The effect of different fishes on biomass of different submerged macrophytes

$3 B)$. 实验结束时所有实验组 TP 浓度都高于对照组, 对照组、鲫组、草鱼组 TP 浓度无显著差异 $(P>0.05)$. 但 鳊组 TP 浓度较高, 与其他 3 组均存在显著差异 $(P>0.05)$. 在整个实验过程中, 所有实验组和对照组中 DTN 浓度呈现逐渐下降的趋势, 实验结束时, 对照组、草鱼组、鳊组和鲫组 DTN 浓度分别为 1.8、2.1、3.0 和 2.7 $\mathrm{mg} / \mathrm{L}$ (图 3C). 实验期间, 所有实验组和对照组 DTP 浓度也呈逐步下降的趋势, 实验结束时, 对照组、草鱼 组、鳊组和鲫组 DTP 浓度分别为 $0.12 、 0.11 、 0.15$ 和 $0.13 \mathrm{mg} / \mathrm{L}$ ( 图 3D).

\section{3 不同鱼类对水体中 Chl.a 浓度的影响}

实验结束时所有实验组 Chl.a 浓度都高于对照组, 表明鱼类的存在会提高水中的 Chl. a 浓度 (图 4). 实 验结束时, 对照组和草鱼组中 Chl. a 浓度分别为 4.5 和 $5.6 \mu \mathrm{g} / \mathrm{L}$, 二者未存在显著差异 $(P>0.05)$. 而鳊组实 验结束时 Chl.a 浓度为 $30.0 \mu \mathrm{g} / \mathrm{L}$, 与对照组、草鱼组存在显著差异 $(P<0.05)$, 鲫组实验结束时 Chl.a 浓度为 $38.0 \mu \mathrm{g} / \mathrm{L}$, 与对照组、草鱼组存在显著差异 $(P<0.05)$.

\section{3 讨论}

研究发现, 草鱼和鳊的存在都不利于沉水植物的生长,其中草鱼的影响力超过鳊, 而鲫的存在有利于沉 水植物的生长与恢复. 研究表明, 草鱼对沉水植物具有很强的摄食作用 ${ }^{[5,7]}$. 鱼类对沉水植物生长的影响主 要通过直接摄食作用以及通过排泄和对沉积物的扰动间接影响沉水植物的生长. 草鱼、鳊都属于草食性鱼 类, 以摄食水生植物为主, 但鳊的咽齿没有草鱼发达, 口裂相比于草鱼也较小, 无法轻易咬断植物纤维 ${ }^{[17]}$, 这 可能是导致鳊对于沉水植物的摄食作用较草鱼弱的原因. 鲫属于杂食性鱼类,除了沉水植物,鲫还可以摄食 水生昆虫、藻类以及其它有机碎屑等作为食物补充. 本实验中未发现有水生昆虫, 所以未发现鲫摄食水生昆 虫. 刘恩生曾报道, 微囊藻是太湖鲫的重要食物之一 ${ }^{[18]}$. 水绵属于藻类中的绿藻门, 本实验中鲫组水绵生物 量显著小于对照组, 表明鲫摄食水绵, 即水绵是鲫的食物之一; 由于未分析藻类组成, 所以不能确定鲫是否 还摄食其它藻类; 金鱼藻和苦草不易被鲫摄食, 但当它们的部分死亡茎叶形成的碎屑可能被鲫摄食, 从而作 为食物来源之一. 另外, 鲫下咽齿只有 1 行, 而鳊下咽齿有 3 行; 鲫和鳊除了下咽齿行数不同以外, 结构也不 

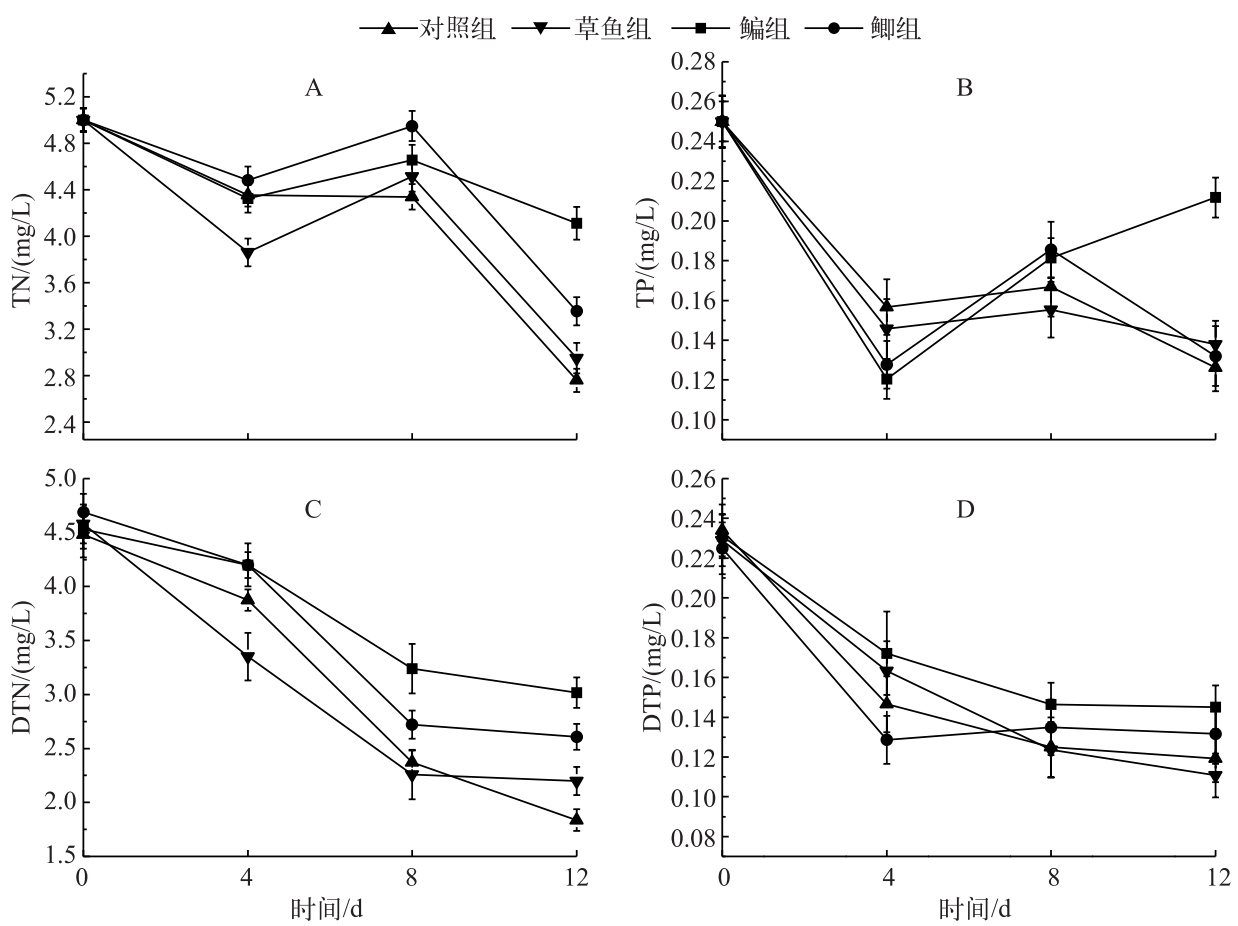

图 3 不同处理组水体中营养盐浓度的变化

Fig.3 Changes of TN,DTN, TP and DTP concentrations in different treatments

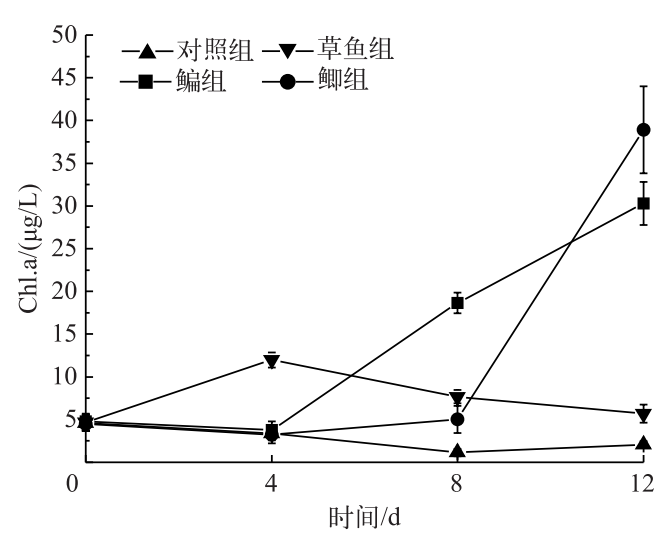

图 4 实验结束时不同处理组 Chl.a 浓度的变化

Fig.4 Changes of Chl.a concentration in different treatments at the end of the experiment
同, 鲫的下咽齿外侧齿略扁, 内侧齿白齿状, 而鳊鱼下 咽齿细长, 尖端弯曲呈钩状, 所以鳊的咽齿比鲫发达, 更容易咬断植物纤维, 这可能是鳊对沉水植物的摄食 能力强于鲫的原因之一. 对沉水植物摄食作用的差异 可能是导致 3 种鱼类对沉水植物的生长影响不同的 原因.

3 种鱼对不同种沉水植物生长的影响不同. 其中, 3 种鱼实验组中金鱼藻的生物量增长最高; 鲫组马来眼 子菜的生物量下降最高; 草鱼组与鳊组伊乐藻的生物 量下降最高. 孙健等研究表明草鱼对轮叶黑藻、苦草、 蕰草、金鱼藻具有选择性摄食, 草鱼最不喜欢摄食的是 金鱼藻 ${ }^{[19]}$. 有研究报道, 鲤鱼等杂食性鱼类也不喜欢摄 食金鱼藻. 金鱼藻与马来眼子菜、伊乐藻、苦草相比较, 叶边缘具有散生的刺状细齿, 虽含有丰富的蛋白成分, 但粗纤维含量、木质素也较高 ${ }^{[7,20]}$, 而不利于鱼的摄食, 这可能是 3 种鱼都不喜欢摄食金鱼藻, 从而导致金鱼藻 的生物量增长率最高的原因. 马来眼子菜粗纤维含量低、营养成分易消化吸收; 有研究报道马来眼子菜根系 不发达, 植株再生能力较弱, 同时粗纤维含量低、营养成分易消化吸收而为草鱼、团头鲂等鱼类所喜 食 ${ }^{[19-21]}$. 这可能是本实验中鲫喜欢摄食马来眼子菜从而导致马来眼子菜的生物量下降率最高的原因. 有研 究表明, 伊乐藻的营养成分易被草鱼、团头魵消化吸收 ${ }^{[22]}$, 所以, 这可能是草鱼与鳊喜欢摄食伊乐藻从而导 致伊乐藻生物量下降率最高的原因. 
3 种鱼类都抑制沉水植物的竞争者一水绵的生长. 水绵是丝状绿藻, 其生长需要光照、营养盐和温度等 条件. 当水绵与沉水植物共同存在时必然与沉水植物竞争光照、营养盐, 从而不利于沉水植物的生长. 有研 究表明, 水绵还可以通过化感作用抑制沉水植物的生长 ${ }^{[23]}$. 研究表明 ${ }^{[17]}$, 木质素、单宁物质等抗营养物质是 影响鱼类适口性的重要因素, 由于水绵缺乏木质素和纤维组织, 藻体柔软而有弹性,这可能是因为 3 种鱼类 都喜欢主动摄食水绵, 从而抑制水绵生长. 在利用沉水植物修复富营养水体时, 可以考虑利用鱼类对水绵的 摄食来控制水绵的生长.

3 种鱼类实验组 TN、TP 和 Chl.a 浓度都高于对照组. 姚洁等 ${ }^{[10]}$ 研究发现罗非鱼的直接牧食导致苦草生 物量显著降低, 同时增加了水中 TN 和 TP 浓度, 浮游植物的生物量 (Chl.a 浓度) 也明显增加. 鱼类增加水中 营养盐浓度的途径包括排泄和对沉积物的扰动 ${ }^{[24-25]}$. 这可以解释为什么本实验结束时所有实验组 TN、TP 浓度都高于对照组. 水体中氮、磷浓度的增加有利于浮游植物的生长, 所以 Chl. a 浓度也会相应的增加, 这也 可以解释为什么 3 种鱼类实验组 Chl. a 浓度都高于对照组. 各实验组 TN、TP 与 DTN、DTP 浓度整个实验期间 变化趋势不一致, 如实验第 $8 \mathrm{~d}$ 所有实验组 TN、TP 浓度都上升, 而 DTN DTP 浓度却下降(图 3), 可能是鱼 类排泄和对沉积物的扰动增加了 $\mathrm{TN} 、 \mathrm{TP}$ 浓度, 但因为鱼类的粪便和沉积物大部分为非溶解性氮、磷, 这可能 是导致 TN、TP 与 DTN、DTP 整个实验期间变化趋势不一致的原因之一.

该研究结果在利用沉水植物进行富营养水体修复中具有参考价值, 例如在沉水植物修复富营养化水体 时, 在沙质和碎石等底质的水域, 可优先考虑投放鲫, 鲫既可以控制水体中水绵生长, 同时又确保沉水植物 不被大量摄食; 而在泥土底质的水域需要考虑鱼类的扰动作用, 尤其在鱼类繁殖季节的扰动作用可能会导 致透明度下降从而影响沉水植物获得光照, 进而影响沉水植物的生长. 同时在利用沉水植物修复水体时, 需 要控制草鱼和鳊的密度, 以避免草鱼和鳊对沉水植物的过量摄食. 在选择沉水植物种类时, 可以优先考量鱼 类相对不喜欢摄食的金鱼藻.

\section{4 参考文献}

[ 1 ] Jeppesen E, Søndergaard M, Sondergaard M et al. The structuring role of submerged macrophytes in lakes. Ecological Studies, 1998, 131: 381-392. DOI: 10.1007/978-1-4612-0695-8.

[ 2 ] Hu Lian, Wan Chenyan, Shen Jianzhong et al. Application of submerged plants in ecological restoration of eutrophic waters. Reservoir Fisheries, 2006, 26(5) : 69-71 (in Chinese with English abstract). [胡莲, 万成炎, 沈建忠等. 沉水植物 在富营养化水体生态恢复中的作用及前景. 水利渔业, 2006, 26(5): 69-71.]

[ 3 ] Moss B. Engineering and biological approaches to the restoration from eutrophication of shallow lakes in which aquatic plant communities are important components. Hydrobiologia, 1990, 200/201: 367-377. DOI: 10.1007/978-94-017-0924-8_31.

[ 4 ] Scheffer M, Hosper SH, Meijer ML et al. Alternative equilibria in shallow lakes. Trends in Ecology \& Evolution, 1993,8 (8) : 275-279. DOI : 10.1016/0169-5347 (93) 90254-M.

[ 5 ] Yang Qingxin. Studies on the interaction of submerged plant and phytoplankton in eutrophic waters. J Lake Sci, 1996, 8 (Z1) : 17-24 (in Chinese with English abstract). DOI: 10.18307/1996.sup03. [ 杨清心. 富营养水体中沉水植物与浮 游藻类相互竞争的研究. 湖泊科学, 1996, 8(Z1): 17-24.]

[ 6 ] Wang Hua, Pang Yong, Liu Shenbao et al. Research progress on influencing of environmental factors on the growth of submersed macrophytes. Acta Ecologica Sinica, 2008, 28(8) : 3958-3968(in Chinese with English abstract). [王华, 逢勇, 刘申宝等. 沉水植物生长影响因子研究进展. 生态学报, 2008, 28(8):3958-3968.]

[ 7 ] Catarino LF, Ferreira MT, Moreira IS. Preferences of grass carp for macrophytes in Iberian drainage channels. Journal of Aquatic Plant Management, 1997, 36: 79-83. DOI: 10.2478/s13545-012-0004-4.

[ 8 ] Hanlon SG, Hoyer MV, Cichra CE et al. Evaluation of macrophyte control in 38 Florida lakes using triploid grass carp. Journal of Aquatic Plant Management, 2000, 38: 48-54.

[ 9 ] Spencer DF. Estimating the impact of triploid grass carp on sago pondweed in the Bymes Canal: Implications for biological control in northern California irrigation systems. Ecological Modelling, 1994, 72(3/4) : 187-204. DOI : 10.1016/03043800 (94) 90083-3.

[10] Yao Jie, Liu Zhengwen. Effects of tilapia on submerged macrophyte Vallisneria spiralis in eutrophic waters. Ecology \& Environmental Sciences, 2010, 19(5) : 1063-1067(in Chinese with English abstract). [姚洁, 刘正文. 罗非鱼对富营养型 
水体中沉水植物苦草的影响. 生态环境学报, 2010, 19(5): 1063-1067.]

[11] Ji Yachan, Zhang Ming, Li Kuanyi et al. Effects of Carp disturbance on Vallisneria natans (Lour. ) Hara. The academic seminar assembly of Fish China Institute of Oceanology and Limnology society, Chinese Animal Society of Ichthyology branch in 2012. 2012 (in Chinese). [姬娅婵, 章铭, 李宽意等. 鲤鱼扰动对苦草生长的影响. 中国海洋湖沼学会鱼 类学分会、中国动物学会鱼类学分会 2012 年学术研讨会论文摘要汇编, 2012.]

[12] Lei Zexiang. Study on aquatic macrophyte vegetations and their environment in Taihu Lake[Dissertation]. Guangzhou: Jinan University, 2006(in Chinese with English abstract). [ 雷泽湘. 太湖大型水生植被及其环境效应研究 [ 学位论文]. 广州: 暨南大学, 2006.]

[13] Qiu Dongru, Wu Zhenbin. On the decline and restoration of submerged vegetation in eutrophic shallow lakes. J Lake Sci, 1997, 9(1) : 82-88( in Chinese with English abstract). DOI: 10.18307/1997.0113. [ 邱东茹, 吴振斌. 富营养化浅水 湖泊沉水水生植被的衰退与恢复. 湖泊科学, 1997, 9(1):82-88.]

[14] Lin Tao, Cui Fuyi, Liu Dongmei et al. Effect of food habits of some fishes on the biomanipulation in drinking water source management. Journal of Harbin Institute of Technology, 2006, 38(1) : 35-37 (in Chinese with English abstract). [ 林涛, 崔福义, 刘冬梅等. 水源治理中鱼类的摄食选择性对其生物操纵作用的影响. 哈尔滨工业大学学报, 2006, 38 (1) : 35-37.]

[15] Jin Xiangcan, Tu Qingying eds. Specifications for lake eutrophication survey. Beijing: China Environmental Science Press, 1990 (in Chinese).[金相灿，屠清瑛. 湖泊富营养化调查规范. 北京: 中国环境科学出版社, 1990.]

[16] Zhang Zongshe, Huang Xiangfei eds. Research methods of freshwater plankton. Beijing: Science Press, 1991: 358-382(in Chinese). [ 章宗涉, 黄祥飞. 淡水浮游生物研究方法. 北京: 科学出版社, 1991: 358-382.]

[17] Feng Deqing, Huang Xiusheng, Tang Longfei et al. Evaluation of forages palatability for grass carp and white bream in mountain area of South China. Chinese Journal of Grassland, 2006, 28(4) : 75-78 (in Chinese with English abstract). [冯德庆, 黄秀声, 唐龙飞等. 草鱼、鳊对南方几种牧草的适口性评价研究. 中国草地学报, 2006, 28(4): 75-78. ]

[18] Liu En'sheng. A study on diet composition of dominant fishes in Lake Taihu. Journal of Fisheries of China, 2008, 32(3): 395-401 (in Chinese with English abstract). DOI: 10.3724/SP.J.00001. [刘恩生. 太湖主要鱼类的食物组成. 水产学 报, 2008, 32(3) : 395-401.]

[19] Sun Jian, He Feng, Zhang Yi et al. The feeding behavior of grass carp (Ctenopharyngoden idellvs) on different types of submerged plants. Acta Hydrobiologica Sinica, 2015, 39(5): 997-1002 (in Chinese with English abstract). DOI: 10. 7541/2015.130. [孙健, 贺锋, 张义等. 草鱼对不同种类沉水植物的摄食研究. 水生生物学报, 2015, 39(5): 997-1002. ]

[20] Zhu Shunqing, Zhou Gang, Yu Neng et al. The transplanting of feeding aquatic plants in Lake Gehu with relation to effectiveness for pen fish farming. Journal of Fisheries of China, 1993, 17(3) : 189-197 (in Chinese with English abstract). [ 朱顺清, 周刚, 于能等. 以滆湖为例湖泊种植饲料水草的养鱼效果研究. 水产学报, 1993, 17(3): 189-197.]

[21] Chen Hongda. Impact of aquaculture on the ecosystem the Donghu Lake, Wuhan. Acta Hydrobiologica Sinica, 1989,13 (4) : 359-368 (in Chinese with English abstract). [ 陈洪达. 养鱼对武汉东湖生态系的影响. 水生生物学报, 1989, 13 (4) : 359-368. ]

[22] Wei Yun, Chen Yuangao, Liu Zhengwen et al. Digestion and utilization of Elodea nuttallii by Ctenopharynyodon idella and Megalobrama amblycephala. J Lake Sci, 1996, 8( Z1) : 63-72(in Chinese with English abstract). DOI : 10.18307/1996. sup08. [魏云，陈源高，刘正文等. 草鱼、团头鲂对伊乐藻的消化与利用. 湖泊科学, 1996, 8(Z1): 63-72.]

[23] Ma Jianmin, Ma Qing, Su Xiuyan et al. Allelopathy of Spirogyra communis on three submerged plants. Journal of Henan Agricultural Sciences, 2011, 40(9) : 70-73(in Chinese with English abstract). [马剑敏, 马顷, 苏秀燕等. 水绵对 3 种 沉水植物的化感作用研究. 河南农业科学, 2011, 40(9): 70-73.]

[24] Vanni MJ, Bowling AM, Dickman EM et al. Nutrient cycling by fish supports relatively more primary production as lake productivity increases. Ecology, 2006, 87(7) : 1696-1709. DOI: 10.1890/0012-9658(2006)87.

[25] Li Xinzhou, Zhang Huaiyu. Application of aquatic plants in ecological restoration of eutrophic waters. Science \& Technology Information, 2009, (24): 117 (in Chinese with English abstract). [李新舟, 张怀宇. 水生植物在富营养化水体治理 中的应用前景. 科技资讯, 2009, (24) : 117.] 